\title{
AN ANN SOLUTION FOR GENERALIZED SIERPINSKI FRACTAL ANTENNA
}

\author{
Anuradha $^{1}$, Shweta Rani ${ }^{2}$, Sushil Kakkar ${ }^{3}$
}

\begin{abstract}
A neural network (NN) based analysis model is developed for locating the operating frequencies of generalized Sierpinski fractal antenna. The developed model can locate the operating frequencies of standard as well as perturbed antennas. The performance of the neural model is validated with simulations as well as with results reported in literature.
\end{abstract}

Index Terms - Fractals, microstrip antennas, multiband antennas, neural networks

\section{INTRODUCTION}

In response to the ever-increasing demands of wideband and ultra-wide band antenna, considerable amount of effort is currently underway to develop multiband antennas. Much has also been achieved in designing dual band antennas [1-3]. Mostly planar type designs are preferred for these structures due to their valuable merits of small size, Low manufacturing cost and conformability. In the era of modern years researchers were able to achieve the multiband property in reconfigurable antennas, in which the different radiating elements of an array are connected together using switches to form groups of elements that resonate at different frequency bands [4-5]. Furthermore, fractal antennas have been used as miniature multiband antenna [6-9] in which the self-similarity property is used to resonate the antenna at a multiple frequency bands. Over the years various fractal structures have been considered for antenna applications. But the largest problem with this category of multiband antennas is that all the resonating frequencies of the antenna in its conventional form are not the user required frequencies. Shifting of the multiple bands of these antennas has been observed by proper control of the scale factor in some fractal structures.

The monopole antenna based on Sierpinski gasket fractal has been studied extensively as an excellent candidate for multiband applications $[10,11]$. The standard structure of this category is the Sierpinski gasket which consists of a series of scaled triangles having a scaling of $\mathrm{s}=0.5$ (scale factor ratio $\delta=2$ ). Its electrical features gets shifts into a $\log$ periodic allocation of frequency bands where these multiple bands each have a common nature. Appearance of this nature has also been observed in the radiation patterns. It has also been demonstrated that the location of the multiple bands can be controlled by proper adjustment of the scale factor used to generate the Sierpinski antenna [12]. Changing the scale factor from 0.5 produces the so called perturbed Sierpinski gasket, which can be used to control the band spacing. The multiband behavior of the antennas, and their radiation patterns for scale factors other than 0.5 were observed in $[12,13]$. However, in all these published results it can be clearly observed that the log periodic nature is compromised for first couple of frequencies and for the rest of the frequencies, the frequency ratios do not exactly match with the scale factor. This deviation is observed for standard as well as perturbed Sierpinski gaskets. Estimated formulas were given for standard and perturbed Sierpinski gaskets, to locate the operational frequencies [11, 14-16]. The formula developed in [14] is applicable for perturbed structures, but this also provides a compromised value for the first band. Authors of [15] have modified the formula developed in [11] for standard Sierpinski gasket.

In the present work, a neuro computational approach is used to locate the operational frequencies of a Sierpinski gasket. The developed network is a general one in the sense that this single network can be used to locate the frequencies of either perturbed or unperturbed gasket of any scale factor $(\mathrm{s}=0.1-0.9)$ and for any gasket height $(\mathrm{h}=10 \mathrm{~mm}$. $-90 \mathrm{~mm}$.). The results of this network have been validated with simulations as well as with earlier reported results. The reason for developing the network is twofold: (i) to reduce the simulation time of analyzing the fractal antenna with the developed CAD model and (ii) during design of the antenna at specified frequencies, the developed model can be put in an optimization loop to find out the structure.

\footnotetext{
a Corresponding author: sonanu8@gmail.com
} 


\section{ANN APPROACH OF GENERALIZED GASKET ANTENNA}

Use of NN for antenna analysis and design is not new and applications have been reported since 1990. In most of the cases, the role of the NN is to form a mapping (blackbox) between a set of input parameters and the corresponding set of outputs [17-21].

In the present work, the characteristics of $\mathrm{NN}$ have been exploited to form a mapping of the set of inputs comprising of overall height (h) of the Sierpinski monopole gasket and the scale factor (s) with set of outputs comprising of $\mathrm{fr} 1, \mathrm{fr} 2 / \mathrm{fr} 1$ and $\mathrm{fr} 3 / \mathrm{fr} 1$, where fr 1 , fr 2 and fr 3 are first, second and third resonant frequencies of a two-iterated generalized Sierpinski gasket. A model of generalized Sierpinski gasket monopole antenna is shown in Figure.1, where the $\mathrm{h}$ defines the entire height of the triangle and $\mathrm{s}$ represents the scale factor. This model is fed at the bottom using the co-axial cable of 50 $\Omega$. The NN model of this antenna is shown in Figure.2. Although the formulation can be generated for higher iteration fractals, but most of the practical antennas are of dual-band type and at the most triple-band type. Furthermore, the purpose of this work is to find the working frequencies of the fractal based multiband antenna, because fractal antennas mostly deviate from their fractal behaviour in lower iterations.

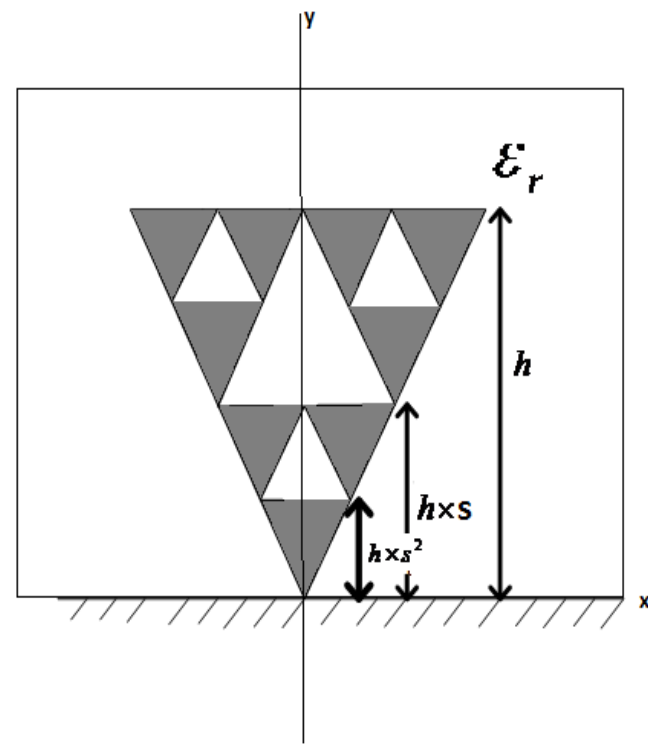

Fig.1 Generalized Sierpinski Monopole Antenna

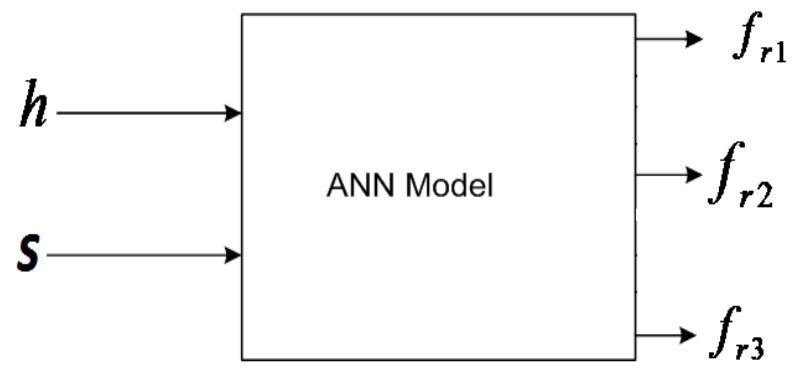

Fig.2 NN Model of Generalized Sierpinski Antenna
Data Generation: For generation of training data for the neural network, a set of skewed gaskets were formed by varying the scale factor. Instead of a similarity transformation, an affine transformation was applied to go from the overall structure to these skewed gaskets (Fig 1). The Iterated function system [10], which represents a versatile method for convenient generation of wide variety of useful fractal structures and is based on application of a series of affine transformation, was used to generate these structures. The height of the gasket was varied between $10 \mathrm{~mm}$. $-90 \mathrm{~mm}$. in steps of $5 \mathrm{~mm}$. and the scale factor was between $0.1-0.9$. Simulations were carried out using CST microwave studio [22] to find out the operational frequency bands of two iterated fractal structures

Neural Network Training: Multilayer Perceptrons with the well known back-propagation training algorithm was used to develop the trained network. Table-1 shows the parameters of the neural network. It is to be noted that, we have not taken permittivity of the substrate $\left(\varepsilon_{\mathrm{r}}\right)$ and its thickness ( $t$ ) as an input variable parameters, since in Sierpinski gaskets the role of the dielectric layer is mostly to give support to the antenna structure. For the generation of training data, the gaskets were assumed to be printed on a dielectric sheet of thickness $t=1.588 \mathrm{~mm}$. and $\varepsilon_{\mathrm{r}}=2.5$. Matlab neural network toolbox was used to perform the training [23].

Table-1: Neural network parameters

\begin{tabular}{|l|l|}
\hline No. of training data set & 153 \\
\hline No. of input neurons & 2 \\
\hline No. of hidden layer neurons & 20 \\
\hline No. of output layer neurons & 3 \\
\hline Learning rate & 0.073 \\
\hline Epochs & 1000 \\
\hline Error tolerance & 0.01 \\
\hline
\end{tabular}

\section{RESULTS AND DISCUSSION}

The trained neural network was tested for its validity with the test data from the simulator as well as with the previously reported results. In order to test the generalization of the network, a set of Sierpinski gaskets of input parameters $\{\mathrm{h}, \mathrm{s}\}=\{88.9 .0 .5\},\{89,0.5\},\{89$, $0.6\}$ and $\{89,0.66\}$ were given as input test parameters to the trained NN. Figure 3 and Figure 4 shows the simulated response of the three resonating frequencies corresponds to those resonating frequencies that were obtained with trained $\mathrm{NN}$ for the given input test parameters. In order to verify these resonating frequencies, it was compared with the reported results as shown in Table. 2. These comparison of the neuromodeler response with that obtained from the simulator for the values of $\mathrm{fr} 1, \mathrm{fr} 2 / \mathrm{fr} 1$ and $\mathrm{fr} 3 / \mathrm{fr} 1$. As 
expected, the first frequency is almost same for all values of scaling, because it depends on the overall height of the antenna. As verified by other authors for scaling equal to and more than 0.5, the antenna maintains its fractal behavior and in this range the ANN results are quite in agreement with the simulation results. For scaling less than 0.5 , as seen from the simulation, and also by ANN, all the resonating frequencies are not fractal frequencies. The neuromodeler performance was also tested against earlier reported results $[10,12]$. The results for different height and scaling of the gasket are shown in Table-2 where a good agreement can be seen. The network was trained to give outputs as fr 1 , fr $2 /$ fr 1 and fr $3 /$ fr 1 for ease of calculating all the frequencies and to further use the outputs directly in any optimization loop [24].

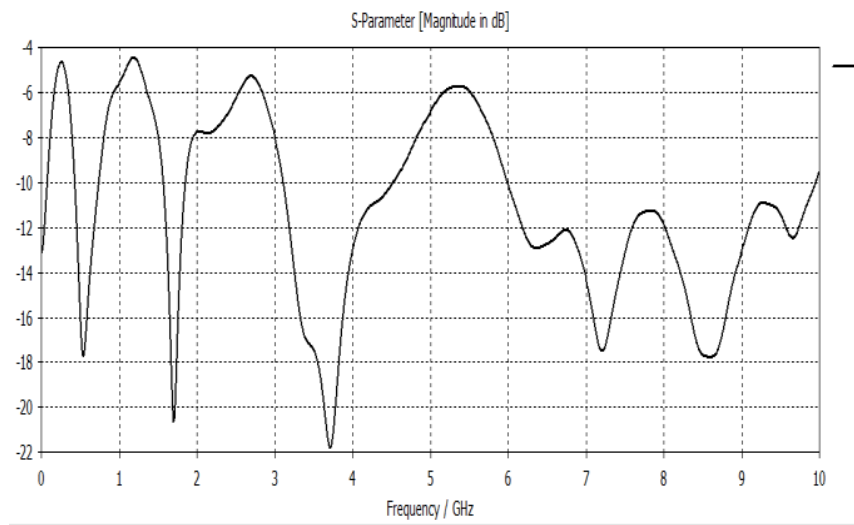

Fig. 3 Simulated response of Sierpinski gasket antenna with design dimensions of $\mathrm{h}=88.9 \mathrm{~mm}, \mathrm{~S}=(0.5)$

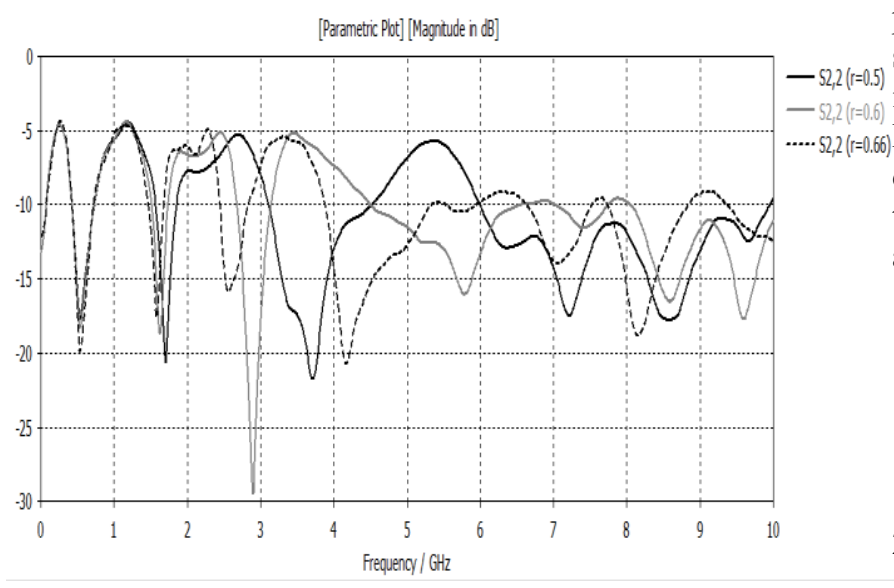

Fig. 4 Simulated parametric response of the Sierpinski gasket antenna with design dimensions of $\mathrm{h}=89 \mathrm{~mm}, \mathrm{~s}=(0.5,0.6,0.66)$
Table:2 Performance comparison of the neuromodeler with earlier reported result

\begin{tabular}{|c|c|c|c|c|c|c|c|}
\hline & \multirow[b]{2}{*}{$\begin{array}{l}\begin{array}{l}\text { Scali } \\
\text { ng }\end{array} \\
(\mathbf{S})\end{array}$} & \multicolumn{3}{|c|}{ Reported Results } & \multicolumn{3}{|c|}{ NN Response } \\
\hline & & $\begin{array}{c}f_{\mathrm{r} 1}(\mathrm{GH} \\
\mathrm{z})\end{array}$ & $\begin{array}{c}f_{\mathrm{r} 2} / f_{\mathrm{r}} \\
1\end{array}$ & $\begin{array}{c}f_{\mathrm{r} 3} / f_{\mathrm{r}} \\
1\end{array}$ & $\begin{array}{c}f_{\mathrm{r} 1}(\mathrm{GH} \\
\mathrm{z})\end{array}$ & $\begin{array}{c}f_{\mathrm{r} 2} / f_{\mathrm{r}} \\
1\end{array}$ & $\begin{array}{c}f_{\mathrm{r} 3} / f \\
\mathrm{r} 1\end{array}$ \\
\hline $\begin{array}{c}\text { Ref. } \\
{[10]} \\
\varepsilon_{\mathrm{r}}= \\
2.5 \\
t= \\
1.588 \\
\text { mm. } \\
h= \\
\mathbf{8 8 . 9} \\
\text { mm. }\end{array}$ & 0.5 & 0.52 & 3.50 & 7.07 & 0.50 & 3.54 & $\begin{array}{c}7.1 \\
0\end{array}$ \\
\hline $\begin{array}{l}\text { Ref. } \\
{[12]}\end{array}$ & 0.5 & 0.46 & 3.77 & 7.64 & 0.48 & 3.60 & $\begin{array}{c}7.6 \\
7 \\
\end{array}$ \\
\hline $\begin{array}{c}\varepsilon_{\mathrm{r}}= \\
2.5\end{array}$ & 0.6 & 0.45 & 3.57 & 6.13 & 0.48 & 3.40 & $\begin{array}{c}5.8 \\
0\end{array}$ \\
\hline $\begin{array}{c}t= \\
1.588 \\
\mathrm{~mm} . \\
h=89 \\
\mathrm{~mm} .\end{array}$ & 0.666 & 0.44 & 3.46 & 5.53 & 0.47 & 3.26 & $\begin{array}{c}5.5 \\
3\end{array}$ \\
\hline
\end{tabular}

\section{CONCLUSION}

Self-similarity is a property common to many fractals but in order to become a useful radiator it is necessary for the fractal antenna to fulfill specific requirements for the desired frequencies. For this reason, a generalized NN model was developed for the analysis of the Sierpinski gasket in order to locate the operational frequencies. The performance of the network was validated both with the simulation and the published results. With the growing interest in using fractals as multiband antenna for GSM, DECT, WLAN, ISM band hand set applications, the developed $\mathrm{NN}$ model can be useful to effectively locate the frequency bands of operation. Similar formulation can also be developed for other fractal structures.

\section{REFERENCES}

1. S. Maci, and G. B. Gentili, "Dual Frequency Patch Antennas," IEEE Antennas and Propagation Magazine, Vol. 39, No. 6, pp. 13-20, December 1997

2. S. C. Gao, L. W. Li, T. S. Yeo, and M. S. Leong, "Small Dual Frequency Microstrip Antennas," IEEE Transactions on Vehicular Technology, Vol. 51, No. 1, pp. 28-36, January 2002.

3. A. Ks. Skrivervik, J. F. Zurcher, O. Staub, and J. R. Mosig, "PCS Antenna Design: The Challenge of Miniaturization," IEEE Antennas and Propagation Magazine, Vol. 43, No. 4, pp. 12- 26, August 2001.

4. J. Ollikainen, M. Fischer, and P. Vainikainen, "Thin Dual-Resonant Stacked Shorted Patch Antenna for Mobile Communications," Electronics Letters, Vol. 35, No. 6, pp. 437-438, March 1999.

5. D. N. Elsheakh, H. A. Elsadek, and E. A. Abdallah, "Reconfigurable Single and Multiband Inset Feed Microstrip Patch Antenna for Wireless Communication Devices," Progress in 
Electromagnetic Research C, Vol. 12, pp. 191-201, 2010.

6. L. Lizzi, and A. Massa, "Dual-band Printed Fractal Monopole Antenna for LTE Applications," IEEE Antennas and Wireless Propagation Letters, Vol. 10, pp. 760- 763, August 2011.

7. J. P. Gianvittorio, and Y. Rahmat-Samii, "Fractals Antennas: A Novel Antenna Miniaturization Technique and Applications," IEEE Antennas and Propagation Magazine, Vol. 44, No. 1, pp. 20- 36, February 2002.

8. D. H. Werner, and S. Ganguly, "An Overview of Fractal Antenna Engineering Research," IEEE Antennas and Propagation Magazine, Vol. 45, No. 5, pp.38-57, February 2003.

9. Anuradha, A. Patnaik, and S. N. Sinha, "An Investigation on E-shaped Fractal Like Patch Antenna," International Symposium on Antenna and Propagation, South Carolina, USA, June 2009.

10. C. Puente, and J. Romeu, "On the behavior of the Sierpinski multiband fractal antenna," IEEE Transactions on Antennas and Propagation., vol. 46, no.4, pp. 517-524, April 1998.

11. C. Puente. Romeu, R.Pous, X.Garcia, and F,Benitez, "Fractal multiband antenna based on the Sierpinski gasket," ElectronLett, vol. 32, no.1, pp. 1-2, Jan 1996.

12. C. Puente, J. Romeu, R.Bartoleme and R.Pous "Perturbation of the Sierpinski antenna to allocate operating bands, Electron.Lett" vol.32, pp. 21862188, Nov. 1996.

13. C.T.P.Song, P.S.Hall, H.Ghafouri-Shiraz, and D.Wake, "Sierpinski monopole antenna with controlled band spacing and input impedance," Electron.Lett. vol. 35, pp. 1036-1038, Jun 1999.

14. C.T.P.Song, P.S.Hall, and H.Ghafouri-Shiraz, "Perturbed Sierpinski Multiband Fractal Antenna with Improved Feeding Technique," IEEE Transactions on Antennas and Propagation, vol51, no.5, May 2003.

15. R.K.Mishra, R.Ghatak, and D.R.Poddar "Design Formula for Sierpinski Gasket Pre-Fractal PlanarMonopole Antennas," IEEE Antennas and Propagation Magazine, vol. 50, no.3, June 2008.

16. J.K Wojciech “ Modified Sierpinski Fractal Monopole for ISM-bands handset Applications", IEEE Transactions on Antennas and Propagation, vol 57, no.3, March 2009.

17. Y. Kim, "Application of Artificial Neural Antennas to Broadband antenna design based on parametric frequency model", IEEE Transactions on Antennas and Propagation., vol. 55, no.3, March 2007.

18. R.K.Mishra and A.Patnaik, "Neural Network-Based CAD Model for the design of Square-patch Antennas", IEEE Transactions on Antennas and Propagation., vol. 46, no.12, Dec 1998.

19. Q. J. Zhang, K. C. Gupta and V. K. Devabhaktuni, "Artificial Neural Networks for RF and Microwave Design - From Theory to Practice," IEEE Trans. Microwave Theory and Techniques, vol. 51, no. 4, 1339-1350, April 2003.
20. R. C. Eberhart and Y. Shi, "Evolving artificial neural networks," in Proc. 1998 Int. Conf. Neural Networks and Brain, Beijing, P.R.C., 1998.

21. Anuradha, A. Patnaik, and S. N. Sinha, "Neurocomputational Analysis of Generalized Koch Fractal Antenna," A Workshop on Advanced Antenna Technology, Puri, Orissa, India, 31 May-4 June 2010.

22. CST Microwave Studio .,http://www.cst.com.

23. www.mathworks.com

24. Anuradha, A. Patnaik, and S. N. Sinha, "Design of Custom Made Fractal

25. Multiband Antennas using ANN-PSO," IEEE Antennas and Propagation Magazine, Vol. 53, No. 4, pp. 94-101, 2011 and [correction] Vol.53, No.5, pp.122- 124, 2011. 